\title{
Complexities for Assessment and Treatment of Co-Occurring ADHD and Tics
}

\author{
Barbara J. Coffey ${ }^{1,2}$
}

Published online: 19 August 2015

(C) Springer International Publishing Switzerland 2015

\begin{abstract}
Bidirectional overlap between attention-deficithyperactivity disorder (ADHD) and tic disorders has long been described. Twenty percent of individuals with ADHD may meet diagnostic criteria for a tic disorder, and more than $60 \%$ of children ages 6-17 with Tourette's disorder (TD) in the community had also been diagnosed with ADHD/ADD. While comorbid presentation of ADHD and tic disorders is firmly established, underlying genetic and pathophysiologic mechanisms need additional investigation. Inhibition is a core deficit in both $\mathrm{ADHD}$ and tic disorders, and research suggests a diffuse process in the brain involving corticostriatothalamicortical (CSTC) pathways in the basal ganglia, striatum, and frontal lobes. The complex presentation of these patients requires comprehensive evaluation and prioritization of treatment goals. Most studies indicate that ADHD places a greater burden on patients than do tics. Optimal patient outcomes may depend on management of both ADHD symptoms and tics when they co-occur. Research in the past decade has shown that these conditions can be safely treated simultaneously.
\end{abstract}

Keywords ADHD · Attention-deficit/hyperactivity disorder · Tics · Tourette's disorder · Treatment $\cdot$ Comorbidity · Corticostriatothalamicortical(CSTC) pathways $\cdot$ Bidirectional overlap

This article is part of the Topical Collection on Tourette's Syndrome

Barbara J. Coffey

Barbara.coffey@mssm.edu

1 Tics and Tourette's Clinical and Research Program, Division of Tics, OCD and Related Problems, Icahn School of Medicine at Mount Sinai, New York, USA

2 Research Psychiatrist, Nathan S. Kline Institute for Psychiatric Research, Orangeburg, NY, USA

\section{Introduction}

\section{Bidirectional Overlap}

Bidirectional overlap between attention-deficithyperactivity disorder (ADHD) and tic disorders has long been described. Both $\mathrm{ADHD}$ and tic disorders are among the neurodevelopmental disorders of symptom onset before age 18 years. ADHD is characterized by a persistent pattern of inattention and/or hyperactivity-impulsivity that interferes with functioning or development with onset before age 12 years. Comorbid psychiatric disorders are very frequent with $\mathrm{ADHD}$, including other disruptive behavior disorders, such as oppositional defiant disorder and conduct disorder, mood, and anxiety disorders. Approximately $20-30 \%$ of patients with ADHD meet criteria for a tic disorder, and ADHD co-occurs in more than $60 \%$ of children with TD ages 6-17 [1]. One population-based study of school age children found comorbid psychiatric disorders in $92 \%$ of the children with TD; ADHD was the most frequent co-occurring condition [2]. As many as half of clinically referred TD patients show signs of ADHD prior to onset of tics, usually preceding the onset of tics by 2 to 3 years $[1,3]$.

To further complicate the clinical phenomenology of TD and ADHD, 20 to $60 \%$ of TD patients meet full criteria for obsessive compulsive disorder (OCD) [4]. OCD symptoms typically emerge at about age 11 to 12 years, after tics have been present for some time. Some studies have reported that as tics improve in early adolescence, OCD symptoms may intensify and often persist. In addition to ADHD and OCD, oppositional defiant disorder, mood disorders, and other non-OCD anxiety disorders have been reported in individuals with TD [4].

Co-occurring disorders confer a more difficult clinical course and outcome [5]. ADHD and OCD often interfere more with overall functioning than do tics. Co-occurring TD 
and ADHD often are associated with academic and peer problems, family conflict, and disruptive behavior. It has been clearly demonstrated that comorbid ADHD and/or OCD leads to greater disability than tic disorders alone [6-10].

\section{Neurobiology}

Given the bidirectional overlap in clinical presentation, it is not surprising that common core symptomatology and putative etiology/pathophysiology have been suggested. Inhibition is a core deficit in both ADHD and tic disorders. Research suggests a diffuse process in the brain involving corticostriatothalamicortical (CSTC) pathways in the basal ganglia, striatum, and frontal lobes with dysregulation involving multiple neurotransmitters. Executive function abnormalities in both are thought to result from fronto-striatal and fronto-parietal network dysfunction. Basal ganglia disinhibition may be intensified by frontal hypoactivity in ADHD. Increased myelination over time may be associated with improvement of tic and ADHD symptoms. Midbrain dopaminergic neurons play a key role in motor control and attentional processes through direct connections to the striatum and cortex, respectively. Dopaminergic pathways and dysregulation play a central role in both tic disorders and ADHD. Higher levels of dopaminergic innervation of the striatum in patients with TD in contrast to healthy controls in single photon emission computed tomography (SPECT) studies have been reported [11]. TD patients have been reported to release more dopamine in response to amphetamine at dopaminergic synapses than healthy controls. Additionally, dopamine-releasing drugs, such as stimulants, may precipitate or exacerbate tics.

Peterson and colleagues reported increased overall hippocampus and amygdala volumes and a decline in volume with age in children and adults with TD compared to healthy controls. In the TD patients, volumes were correlated inversely with tic, OCD, and ADHD severity. These subregions may play a neuromodulatory and compensatory role with tic, OCD, and ADHD symptoms [12]. In addition, cerebral hyperintensities on $\mathrm{T} 2$-weighted magnetic resonance imaging suggestive of neuroinflammatory or neurodegenerative processes have been detected at increased rates in youth with $\mathrm{TD}, \mathrm{OCD}$, or ADHD in subcortical regions (primarily basal ganglia and thalamus). This finding supports a role of subcortical damage and dysfunction in the pathophysiology of these conditions [13].

Both ADHD and TD are conceptualized as inherited neurodevelopmental conditions. Twin studies show 50-90 \% concordance for tic disorders in monozygotic pairs, in contrast to about $20 \%$ concordance among dizygotic twins [14]. Other studies have confirmed a higher percentage of tic disorders in first-degree relatives of TD patients than those of normal controls. Current understanding of TD suggests multiple susceptibility genes; however, a recent GWAS study reported by the
Tourette Syndrome Association International Consortium for Genetics reported that no genes reached threshold significance [15]. Although the underlying molecular-genetic foundation for ADHD has not been elucidated, family, twin, and adoption studies suggest a strong genetic component. Candidate gene associations of minor effect size have been replicated across a number of genes including SLC6A3, DRD5, DRD4, SLC6A4, LPHN3, SNAP-25, HTR1B, NOS1, and GIT1 [16]. A recent study reported that the KCNJ5 gene is associated with both ADHD and TD [17].

In addition, environmental factors may contribute to the pathophysiology and clinical manifestations of both TD and ADHD. Prenatal and perinatal risk factors implicated in both ADHD and TD include alcohol use and smoking during pregnancy, prematurity, and low birth weight $[18 \bullet \cdot]$. One study reported that children with both ADHD and TD had greater likelihood of low birth weight, prematurity, breathing problems, and maternal smoking compared to children with TD only [19]. In a retrospective study, Motlagh and coworkers compared heavy maternal smoking and severe psychosocial stress during pregnancy in youth with TD alone, ADHD alone, TD plus ADHD, or neither. All clinical groups demonstrated higher rates of heavy maternal smoking and severe psychosocial stress during pregnancy than controls, but the difference was statistically significant only for the ADHD alone group [20].

\section{Course and Outcome}

Practitioners often find it challenging to disentangle symptoms and impact of ADHD from tics in the phenomenology and clinical presentation of patients with this comorbid picture. For example, it is not entirely clear whether children with comorbid Tourette's disorder and ADHD have two separate disorders or one complex syndrome with features of both. Course and outcome of ADHD and tic disorders have similarities and differences. ADHD symptoms typically onset before tics, in the preschool or early school age period, and tend to persist in the long run. Tics typically onset in the early school age period, and frequently attenuate or remit later in adolescence. In children and adults with ADHD, comorbid tic disorders do not appear to significantly impact outcome, as ADHD is typically the more impairing disorder. Spencer and colleagues, in a study of 128 boys with ADHD and 110 healthy controls followed over 4 years, reported that $34 \%$ of youth with ADHD had or developed tics; while the remission rate for tics was $65 \%$, in contrast, the remission rate for ADHD was only $20 \%$. The tic remission rate was independent of ADHD, and tic disorders did not significantly impact the course of ADHD [21]. Additionally, in a similar study of adults, those with ADHD had higher lifetime rates of tic disorders than controls, and the tics did not impact the course of ADHD [22]. 
However, when evaluated from the perspective of TD patients with ADHD, evidence suggests that ADHD confers a more difficult clinical course and outcome than for those with TD alone [23]. Co-occurring TD and ADHD in children are often associated with academic problems, peer and family conflicts, and disruptive behavior. It has been clearly demonstrated that comorbid ADHD and/or OCD leads to greater disability than tic disorders alone [6-10]. Data from the Tourette Syndrome International Database Consortium of more than 6000 cases indicated that TD plus ADHD was associated with an earlier TD diagnosis, and a much higher rate of anger control problems and oppositional defiant disorder, sleep difficulties, learning disability, mood disorders, social skills deficits, sexually inappropriate behavior, and selfinjurious behavior than TD alone [24]. In another study, parent ratings of aggression and delinquent behavior and teacher ratings of conduct problems were elevated in those with ADHD alone and those with ADHD and TD [8]. Rizzo and colleagues compared behavioral phenotypes in four groups of 80 youth ages 6-16 years: TD alone, ADHD alone, TD + ADHD, and controls. Results indicated that all clinical groups differed significantly from controls, and ADHD with or without TD was associated with more behavioral problems and lower IQ. Those with TD alone did not differ from controls in behavioral ratings or IQ [25]. Similarly, in a study of 80 adults with TD with or without ADHD, those with ADHD had significantly more depression, anxiety, $\mathrm{OCD}$, and behavioral problems than those without ADHD [26]. Carter and coinvestigators found that children with TD and ADHD experienced more emotional and behavioral problems and more difficulty with social adaption than control subjects and those with TD alone [27]. Other research examining the impact of tics on the course and prognosis of ADHD $[28,29]$ demonstrated that co-occurrence of these disorders leads to a more complicated course and outcome.

Gorman and colleagues examined psychosocial functioning in late adolescents with TD compared to age-matched peers. Compared with controls, those with TD had higher rates of ADHD, depression, learning, and conduct disorders. TD subjects had poorer psychosocial outcomes, which were associated with greater ADHD, OCD, and tic severity [23]. These findings are supported by numerous studies [30].

Older patients with TD and comorbid disorders are also reported to experience a more severe course. Adults with TD and ADHD have been reported to experience significantly more depression, anxiety, obsessive-compulsive symptoms, and maladaptive behaviors than adults with TD only [26].

\section{Evaluation}

Onset of ADHD symptoms, typically in preschool or the early school age period, usually precedes onset of tics which typically occurs at around age 6-7 years. Especially if hyperactivity and impulsivity are present, tics may be overlooked during clinical evaluation. Diagnosis is based on a detailed history of ADHD and tic symptoms. Tics may be suppressed during initial evaluation. For the most part, neurological, laboratory, and neuroimaging findings are within normal limits. Baseline laboratory examination, including complete blood count ( $\mathrm{CBC}$ ), thyroid function tests comprehensive metabolic and lipid panel, and urine toxicology screen, is helpful in the evaluation process to rule out possible underlying medical conditions or substance abuse.

Careful history, physical examination, and review of medical, psychological, and educational records are necessary. A meticulous history of the onset and course of tics should be taken, including response to previous treatments. Use of standardized rating scales such as the Yale Global Tic Severity Scale (YGTSS) for quantitative assessment of tics is helpful in determination of the treatment approach. Domains of inquiry include tic number, frequency, intensity, complexity and interference, and an overall assessment of tic-related impairment [27].

Psychiatric assessment of patients with ADHD and tics should include systematic review of comorbid disorders, including obsessive compulsive disorder (OCD), anxiety, and mood disorders. The Achenbach Child Behavior Checklist (CBCL), Swanson, Nelson and Pelham (SNAP-IV), Children's Yale-Brown Obsessive-Compulsive Scale (CYBOCS), Multidimensional Anxiety Scale (MASC), and Children's Depression Rating Scale (CDRS) are considered gold standard quantitative assessments [28-32].

\section{Treatment}

Treatment of ADHD and tic disorders is based on comprehensive evaluation of the impact of tics, in the context of ADHD, on social, emotional, family, and academic/occupational functioning. Specific practice guidelines for treatment of ADHD and treatment of tic disorders have been developed by the American Academy of Child and Adolescent Psychiatry [33, 34•]. In general, ADHD is more likely to be associated with both short-term and, left untreated, longer-term problems for patients. Many children with ADHD and tics will experience attenuation or remission of their tics as they grow older. If tics are not causing significant distress or impairment, treatment of tics themselves may not be necessary. Mild to moderate tics may only require support and monitoring. Treatment is indicated when tics cause distress or impairment.

The first step of treatment is psychoeducation. Clarification that the tics are involuntary is critical. Advocacy for an optimal school/occupational environment is often necessary. Support groups, such as the Tourette Association of America (http://www.tsa-usa.org), are a valuable resource. 


\section{Pharmacotherapy}

Pharmacotherapy is tailored to the specific needs of each patient. Evaluation of the impact of both ADHD and tics on the patient's overall functioning and quality of life is essential. ADHD symptoms are usually more impairing than tics, and treatment should target the most impairing symptoms first. When tics are treated, the goal of intervention is not elimination, but relief of tic-related impairment and/or distress, and management of tics that allows the patient to function as normally as possible.

\section{Stimulants}

Stimulants have a large and longstanding database supporting their efficacy in youth and adults with ADHD [35]. That said, the use of stimulants for ADHD symptoms in tic disorder patients has been controversial in the past. FDA recommendations still caution against their use in individuals with tics since publication of a persuasive case series in the early 1980s [36]. However, data has accumulated over the last two decades, demonstrating that stimulants are both effective and safe for ADHD symptoms in patients with tics $[37,38]$.

The only head to head comparison study of methylphenidate and dextroamphetamine in youth with ADHD and TD demonstrated that the majority experienced improvement of ADHD symptoms with acceptable tic effects. High doses of dextroamphetamine exacerbated tics in some subjects, whereas relatively high doses of methylphenidate did not [39].

A contemporary and critical view is that there is no evidence to suggest significant risk of onset or exacerbation of tics during treatment with stimulants in patients with ADHD and tics. An important confound in clinical evaluation and treatment is that onset of tics tends to occur at the time when children are first treated with stimulants. Indeed, some patients may experience at least a transient increase in tics when first started on a stimulant. In most cases, tics will return to their previous level over time, especially if medication is started at a low dose and titrated gradually upward. Thus, stimulants may be used judiciously in the treatment of ADHD in the context of tic disorders with careful monitoring.

Bloch and colleagues conducted a meta-analysis of pharmacotherapy treatment for youth with ADHD and tic disorders. Methylphenidate, $\alpha-2$ agonists, desipramine, and atomoxetine were found to be effective for ADHD symptoms with comorbid tics. Supra-therapeutic doses of dextroamphetamine were reported to worsen tics, but therapeutic doses did not. There was no evidence that methylphenidate exacerbated tics in the short term [40].
$\alpha$-Adrenergic Agonists

Guanfacine and clonidine are $\alpha$-adrenergic agonists and considered first line, but off label, pharmacotherapy for mild to moderate tics in the context of ADHD; these medications are also effective for ADHD symptoms. Low-dose $\alpha$-adrenergic agonists produce presynaptic noradrenergic effects that reduce tics, impulsivity, hyperactivity, and inattention. Clonidine was examined in a retrospective chart review over a 4-year period in children with ADHD with and without tic disorders and resulted in improvement of both ADHD and tic symptoms in a significant majority of patients [41]. Guanfacine activates postsynaptic prefrontal $\alpha$-adrenergic cortical receptors and is also efficacious for hyperactivity, impulsivity, and tics [42-44].

Both medications are available in short- and long-acting formulations. Both guanfacine and clonidine are approved for treatment of ADHD in youth and adults. There is established evidence for the efficacy of the short-acting $\alpha$ agonists for tic disorders and for the efficacy of long-acting agents for ADHD, but there is not yet evidence for the efficacy of long-acting agents for tics. Clonidine is also available in a transdermal patch form that may be applied for up to a 1-week period. A 4-week controlled trial in 437 youth demonstrated that transdermal clonidine was more effective than a placebo adhesive patch and well tolerated [45]. A recent meta-analysis of randomized controlled trials in treatment of chronic tic disorders and examination of moderators revealed a significant moderating effect of comorbid ADHD [46••].

Adverse effects include sedation, hypotension, headache, dry mouth, mid-sleep awakening, and dysphoria. Sedation often dissipates over a few weeks. Hypotension is unlikely at the low doses used, but blood pressure, heart rate, and EKG should be monitored at baseline and follow-up. Rebound hypertension, anxiety, and tics are risks if medication is abruptly discontinued.

\section{Atomoxetine}

Atomoxetine, a selective norepinephrine reuptake inhibitor, has been evaluated in youth with ADHD and chronic tics. Studies have reported that atomoxetine improved both ADHD symptoms and tics in youth with ADHD and tics. Adverse effects included tachycardia, nausea, and decreased appetite and weight $[47,48]$.

\section{Tricyclic Antidepressants}

Desipramine has documented efficacy in treatment of tics in patients with ADHD. One retrospective chart review of children with tics and ADHD showed significant improvement of both tics and ADHD symptoms in a majority of patients without major adverse effects [49]. Spencer and colleagues in a 
controlled study found that desipramine was superior to placebo in the treatment of tics and ADHD symptoms [50]. A controlled trial comparing clonidine and desipramine in children with ADHD and TD demonstrated superior efficacy of desipramine over clonidine for ADHD symptoms, and neither exacerbated nor significantly improved tics [51]. Although desipramine has proven efficacy, it is not considered a firstline medication due to potential cardiovascular effects [52]. Careful cardiac monitoring is necessary.

\section{Neuroleptics (Antipsychotics)}

Although first generation neuroleptics haloperidol and pimozide, and most recently, aripiprazole, are the only FDAapproved medications for Tourette's disorder, these agents are generally only used for treatment of moderate to severe tics unresponsive to behavioral interventions or $\alpha$-adrenergic agonists. The neuroleptics act through blockade of D2 (dopamine) receptors in the basal ganglia. Neuroleptics are generally not recommended for treatment of comorbid ADHD symptoms in the context of tic disorders, despite their frequent use in ADHD without tic disorders, most often for their effectiveness in reducing aggressive behavior [53]. When used in patients with ADHD and tics, neuroleptics may primarily impact ADHD symptoms through reduction of motor symptoms.

Controlled trials have demonstrated efficacy for haloperidol, pimozide, and aripiprazole in tic treatment. However, adverse effects are considerable; most problematic are the extrapyramidal effects, including the potential for tardive dyskinesia. These medications are currently recommended only for patients with debilitating tics.

The second-generation antipsychotics (SGA) block both D2 receptors and 5-HT2 receptors. Studies in TD patients have demonstrated comparable efficacy of risperidone, pimozide, and clonidine [54]. The SGAs tend to cause fewer extrapyramidal adverse effects compared to first-generation neuroleptics. Common adverse effects include weight gain, metabolic abnormalities, and fatigue. Ziprasidone, which is typically least likely among the SGAs to be associated with weight gain, was found to be as efficacious as risperidone for tics in one placebo-controlled trial [55]. Because QTc prolongation may occur, EKG monitoring is recommended. Most recently, in a randomized, double blind placebo-controlled study in 61 children ages 6-18 years, aripiprazole, a novel SGA with partial dopamine agonist-antagonist effects was found to be more effective than placebo in reduction of tics [56].

Metabolic and extrapyramidal effects are common to all neuroleptic agents. Fasting blood glucose and lipid panel are recommended before treatment initiation, at maintenance dose, and during regular follow-up. Weight, waist circumference, and body mass index (BMI) should be monitored.
Education, exercise, and nutritional consultation are recommended.

\section{Alternatives}

When established treatments are unsuccessful, alternatives may be considered. Omega 3 fatty have shown some promise $[57,58]$. In a controlled study of pramipexole in children and adolescents with Tourette's disorder, in which there was no significant difference between active drug and placebo, ADHD positively moderated treatment response [59]. Surgical intervention, including deep brain stimulation, is another option, but is currently recommended only for older adolescents or adults with intractable and highly debilitating tics [60].

\section{Behavior Therapy for Tics}

Comprehensive behavioral intervention for tics (CBIT), including habit reversal training or therapy (HRT), is a specific behavioral treatment for tics that has been shown to be as efficacious as pharmacotherapy. Research has demonstrated the superiority of CBIT/HRT over supportive therapy and psychoeducation alone in treatment of tics in both youth and adults $[61,62]$. CBIT/HRT is now considered first-line treatment for mild to moderate tics when intervention is warranted. Treatment components include awareness training, competing response training, relaxation training, contingency management, social support, and relapse prevention. Teaching patients a competing response to target tics to prevent emergence is a central feature. Studies of CBIT in patients with tics and ADHD are needed.

\section{Conclusion}

In summary, ADHD and tic disorders commonly co-occur, and symptoms frequently overlap and are difficult to disentangle. As neurodevelopmental disorders, both ADHD and tic disorders share common neurobiological underpinnings, phenomenology, and frequent comorbidity. Course and outcome share similarities and differences as a function of individual clinical features and symptom complexity. A systematic approach to evaluation of children with ADHD and tic disorders is needed to appropriately target and prioritize symptoms for treatment. Optimal patient outcomes depend on management of both ADHD symptoms and tics when they co-occur. Recent research has demonstrated safe treatment of both conditions simultaneously.

Acknowledgments Barbara J. Coffey would like to thank Julia Case and Zoey Shaw for their assistance in preparation of the manuscript. 


\section{Compliance with Ethics Guidelines}

Conflict of Interest Barbara J. Coffey has received research support for clinical trials in the past 12 months from Astra Zeneca; Auspex; Catalyst; Neurocrine; Otsuka and Shire.

Human and Animal Rights and Informed Consent This article contains studies with human or animal subjects performed by the author.

\section{References}

Papers of particular interest, published recently, have been highlighted as:

- Of importance

•- Of major importance

1. Centers for Disease Control and Prevention. Prevalence of diagnosed tourette syndrome in persons aged 6-17 years-United States, 2007. MMWR. 2009;58(21):581-5.

2. Khalifa N, Von Knorring AL. Psychopathology in a Swedish population of school children with tic disorders. J Am Acad Child Adolesc Psychiatry. 2006;45(11):1346-53.

3. Spencer T, Biederman J, Wilens T. Attention-deficit/hyperactivity disorder and comorbidity. Ped Clin North Am. 1999;46(5):915-27.

4. Kurlan R, Como PG, Miller B, et al. The behavioral spectrum of tic disorders: a community-based study. Neurology. 2002;59(3):414 20.

5. Debes NM, Hjalgrim H, Skov L. The presence of comorbidity in Tourette syndrome increases the need for pharmacological treatment. J Child Neurol. 2009;24(12):1504-12.

6. Storch EA, Merlo LJ, Lack C, et al. Quality of life in youth with Tourette's syndrome and chronic tic disorder. J Clin Child Adolesc Psychol. 2007;36(2):217-27.

7. Rizzo R, Gulisano M, Cali PV, et al. Long term clinical course of Tourette syndrome. Brain Dev. 2012;34(8):667-73.

8. Sukhodolsky DG, Landeros-Weisenberger A, Scahill L, Leckman JF, Schultz RT. Neuropsychological functioning in children with Tourette syndrome with and without attention-deficit/hyperactivity disorder. J Am Acad Child Adolesc Psychiatry. 2010;49(11):115564.

9. Lebowitz R, Motlagh MG, Katsovich L, et al. Tourette syndrome in youth with and without obsessive compulsive disorder and attention deficit hyperactivity disorder. Europ Child Adolesc Psychiatr. 2012;21(8):451-7.

10. Budman CL, Bruun RD, Park KS, et al. Explosive outbursts in children with Tourette's disorder. J Am Acad Child Adolesc Psychiatry. 2000;39(10):1270-6.

11. Wolf SS, Jones DW, Knable MB, et al. Tourette syndrome: prediction of phenotypic variation in monozygotic twins by caudate nucleus D2 receptor binding. Science. 1996;273:1225-7.

12. Peterson BS, Choi HA, Hao X, et al. Morphologic features of the amygdala and hippocampus in children and adults with Tourette syndrome. Arch Gen Psychiatry. 2007;64(11):1281-91.

13. Amat JA, Bronen RA, Saluja S, et al. Increased number of subcortical hyperintensities on MRI in children and adolescents with Tourette's syndrome, obsessive-compulsive disorder, and attention deficit hyperactivity disorder. Am J Psychiatry. 2006;163(6):11068.

14. Price RA, Kidd KK, Cohen DJ, et al. A twin study of Tourette syndrome. Arch Gen Psychiatry. 1985;42:815-20.

15. Scharf JM, Yu D, Mathews CA, et al. Genome-wide association study of Tourette's syndrome. Mol Psychiatry. 2013;18(6):721-8.
16. Hawi Z, Cummins TD, Tong J, et al. The molecular genetic architecture of attention deficit hyperactivity disorder. Mol Psychiatry. 2015;20(3):289-97.

17. Gomez L, Wigg K, Zhang K, et al. Association of the KCNJ5 gene with Tourette syndrome and attention-deficit/hyperactivity disorder. Genes Brain Behav. 2014;13(6):535-42.

18.• Malhany NEI, Gulisano M, Rizzo R, et al. Tourette syndrome and comorbid ADHD: causes and consequences. Eur J Pediatr. 2015;174(3):279-88. A comprehensive review of prevalence, neurobiology, genetics, and treatment of Tourette's Disorder and ADHD.

19. Pringsheim T, Lang A, Kurlan R, et al. Understanding disability in Tourette syndrome. Dev Med Child Neurol. 2009;51(6):468-72.

20. Motlagh MG, Katsovich L, Thompson N, et al. Severe psychosocial stress and heavy cigarette smoking during pregnancy: an examination of the pre- and perinatal risk factors associated with ADHD and Tourette syndrome. Eur Child Adolesc Psychiatry. 2010;19(10):755-64.

21. Spencer T, Biederman J, Coffey BJ, et al. The four-year course of tic disorders in boys with attention deficit hyperactivity disorder. Arch Gen Psychiatry. 1999;56:842-7.

22. Spencer TJ, Biederman J, Faraone $\mathrm{S}$, et al. Impact of tic disorders on ADHD outcome across the life cycle: findings from a large group of adults with and without ADHD. Am J Psychiatry. 2001;158:611-7.

23. Gorman DA, Thompson N, Plessen KJ, et al. Psychosocial outcome and psychiatric comorbidity in older adolescents with Tourette syndrome: controlled study. Br J Psychiatry. 2010;197(1):36-44.

24. Freeman RD. Tourette Syndrome International Database Consortium. Tic disorders and ADHD: answers from a worldwide clinical dataset on Tourette syndrome. Eur Child Adolesc Psychiatry 2007;Suppl 1:15-23. Erratum in: Eur Child Adolesc Psychiatry 2007;16(8):536.

25. Rizzo R, Curatolo P, Gulisano M, et al. Disentangling effects of Tourette syndrome and ADHD on cognitive and behavioral phenotypes. Brain Dev. 2007;29:413-20.

26. Haddad AD, Umoh G, Bhatia V, et al. Adults with Tourette's syndrome with and without attention deficit hyperactivity disorder. Acta Psychiatr Scand. 2009;120(4):299-307.

27. Yale Child Study Center. YGTSS: Yale Global Tic Severity Scale [Measurement instrument]. 1992.

28. Achenbach T. Manual for the child behavior checklist/2-3 and 1992 Profile [Measurement instrument]. 1992.

29. Swanson, JM. SNAP-IV: Swanson, Nolan and Pelham teacher and parent rating scale [Measurement instrument]. 2013.

30. CYBOCS: Children's Yale-Brown obsessive-compulsive scale [Measurement instrument]. 1989.

31. March JS, Parker JD, Sullivan K, Stallings P, Conners KC. The Multidimensional Anxiety Scale for Children (MASC): factor structure, reliability, and validity. J Am Acad Child Adolesc Psychiatry. 1997;36(4):554-65.

32. Poznanski EO, Grossman JA, Buchsbaum Y, Banegas M, Freeman L, Gibbons R. Preliminary studies of the reliability and validity of the children's depression rating scale. J Am Acad Child Adolesc Psychiatry. 1984;23(2):191-7.

33. Pliszka S, The AACAP. Work group on quality issues. practice parameter for the assessment and treatment of children and adolescents with attention-deficit/hyperactivity disorder. J Am Acad Child Adolesc Psychiatry. 2007;46(7):894-921.

34. Murphy T, Lewin A, Storch E, Stock S. Practice parameter for the assessment and treatment of children and adolescents with tic disorders. J Am Acad Child Adolesc Psychiatry. 2013;52(12):134159. Recent review and guidelines by the American Academy of Child and Adolescent Psychiatry for treatment of Tic Disorders, including comorbid disorders such as ADHD. 
35. Faraone S, Buitelar J. Comparing the efficacy of stimulants in ADHD in children and adolescents using meta-analysis. Eur Child Adolesc Psychiatry. 2010;19(4):353-64.

36. Lowe TL, Cohen DJ, Detlor J, et al. Stimulant medications precipitate Tourette's syndrome. JAMA. 1982;247(12):1729-31.

37. Gadow KD, Sverd J, Sprafkin J, et al. Efficacy of methylphenidate for attention-deficit hyperactivity disorder in children with tic disorder. Arch Gen Psychiatry. 1995;52(6):444-55.

38. Tourette's Syndrome Study Group. Treatment of ADHD in children with tics: a randomized controlled trial. Neurology. 2002;58(4): 527-36.

39. Castellanos X, Giedd J, Elia J, et al. Controlled stimulant treatment of ADHD and comorbid Tourette's syndrome: effects of stimulant and dose. J Am Acad Child Adolesc Psychiatry. 1997;36:589-96.

40. Bloch MH, Panza KE, Landeros-Weisenberger A, et al. Meta-analysis: treatment of attention-deficit/hyperactivity disorder in children with comorbid tic disorders. J Am Acad Child Adolesc Psychiatry. 2009;48(9):884-93.

41. Steingard R, Biederman J, Spencer T, et al. Comparison of clonidine response in the treatment of attention-deficit hyperactivity disorder with and without comorbid tic disorders. J Am Acad Child Adolesc Psychiatry. 1993;32(2):350-3.

42. Scahill L, Erenberg G, Berlin Jr CM, et al. Tourette Syndrome Association Medical Advisory Board: Practice Committee. contemporary assessment and pharmacotherapy of Tourette syndrome. NeuroRx. 2006;3(2):192-206. Review.

43. Boon-yasidhi V, Kim YS, Scahill L. An open-label, prospective study of guanfacine in children with ADHD and tic disorders. J Med Assoc Thai. 2005;88(8):S156-62.

44. Chappell PB, Riddle MA, Scahill L, et al. Guanfacine treatment of comorbid attention-deficit hyperactivity disorder and Tourette's syndrome: preliminary clinical experiences. J Am Acad Child Adolesc Psychiatry. 1995;34:1140-6.

45. Du YS, Li HF, Vance A, Zhong YQ, Jiao FY, Wang HM, et al. Randomized double-blind, multicentre placebo-controlled clinical trial of the clonidine adhesive patch for the treatment of tic disorders. Aust N Z J Psychiatry. 2008;42(9):807-1.

46.• Weisman H, Qureshi I, Leckman J, Scahill L, Bloch M. Systematic review: pharmacological treatment of tic disorders: efficacy of antipsychotic and alpha 2 agonist agents. Neurosci Biobehav R. 2013;37: 1162-71. Comprehensive meta-analysis of antipsychotic and alpha agonist medications in treatment of tic disorders. Results demonstrated significant benefit of both medication classes, but indicated ADHD moderates response to alpha-agonists.

47. Allen AJ, Kurlan RM, Gilbert DL, et al. Atomoxetine treatment in children and adolescents with ADHD and comorbid tic disorders. Neurology 2005;27;65(12):1941-9.

48. Spencer TJ, Sallee FR, Gilbert DL, et al. Atomoxetine treatment of ADHD in children with comorbid Tourette syndrome. J Atten Disord. 2008;11(4):470-81.
49. Spencer T, Biederman J, Kerman K, et al. Desipramine treatment of children with attention-deficit hyperactivity disorder and tic disorder or Tourette's syndrome. J Am Acad Child Adolesc Psychiatry. 1993;32(2):354-60.

50. Spencer T, Biederman J, Coffey B, et al. A double-blind comparison of desipramine and placebo in children and adolescents with chronic tic disorder and comorbid attention-deficit/hyperactivity disorder. Arch Gen Psychiatry. 2002;59:649-56.

51. Singer HS, Brown J, Quaskey S, et al. The treatment of attentiondeficit hyperactivity disorder in Tourette's syndrome: a doubleblind placebo-controlled study with clonidine and desipramine. Pediatrics. 1995;95(1):74-81.

52. Biederman J, Baldessarini RJ, Wright V, et al. A double-blind placebo controlled study of desipramine in the treatment of ADD: I. Efficacy. J Am Acad Child Adolesc Psychiatry. 1989;28(5):77784.

53. Olfson M, King M, Schoenbaum M. Treatment of young people with antipsychotic medications in the United States. JAMA Psychiatry 2015 Jul 1. doi: 10.1001/jamapsychiatry.2015.0500.

54. Bruggeman R, Van der Linden C, Buitelaar JK, et al. Risperidone versus pimozide in Tourette's disorder: a comparative double-blind parallel-group study. J Clin Psychiatry. 2001;62(1):50-6.

55. Sallee FR, Kurlan R, Goetz CG, et al. Ziprasidone treatment of children and adolescents with Tourette's disorder: a pilot study. J Am Acad Child Adolesc Psychiatry. 2000;39:29299.

56. Yoo HK, Joung YS, Lee JS, et al. A Multicenter, randomized, double blind, placebo controlled study of Aripiprazole in children and adolescents with Tourette's Disorder. J Clinical Psych 2013;74(8)(9):e772-80.

57. Gabbay V, Babb JS, Klein RG, et al. A double-blind, placebocontrolled trial of $\omega-3$ fatty acids in Tourette's disorder. Pediatrics. 2012;129(6):e1493-500.

58. Bloch M, Qawasmi A. Omega-3 fatty acid supplementation for the treatment of children with attention-deficit/hyperactivity disorder symptomatology: systematic review and meta-analysis. J Am Acad Child Adolesc Psychiatr. 2011;50(10):991-1000.

59. Kurlan R, Crespi G, Coffey B, et al. Pramipexole for TS trial investigators. a multicenter randomized placebo-controlled clinical trial of pramipexole for Tourette's syndrome. Mov Disord. 2012;27(6): 775-8.

60. Neuner I, Podoll H, Janouschek H, et al. From psychosurgery to neuromodulation: deep brain stimulation for intractable Tourette syndrome. World J Biol Psychiatry. 2009;10(4 Pt 2):366-76.

61. Azrin NH, Nunn RG. Habit-reversal: a method of eliminating nervous habits and tics. Behav Res Ther. 1973;11(4):619-28.

62. Piacentini J, Woods DW, Scahill L, et al. Behavior therapy for children with Tourette disorder: a randomized controlled trial. JAMA. 2010;303(19):1929-37. 